\title{
HIGH SPECTRAL RESOLUTION LIDAR BASED ON QUAD MACH ZEHNDER INTERFEROMETER FOR AEROSOLS AND WIND MEASUREMENTS ON BOARD SPACE MISSIONS
}

\author{
Jean-François Mariscal ${ }^{1 *}$, Didier Bruneau ${ }^{1}$, Jacques Pelon ${ }^{1}$, Mathilde Van Haecke ${ }^{1}$, Frédéric
} Blouzon $^{2}$, Franck Montmessin ${ }^{1}$, Hélène Chepfer ${ }^{3}$

${ }^{1}$ LATMOS/IPSL, UVSQ Univ. Versailles St-Quentin, UPMC Univ. Paris 06, CNRS, Guyancourt, France, *jean-francois.mariscal@latmos.ipsl.fr

${ }^{2}$ CNRS/INSU, Division Technique, Meudon, France

${ }^{2}$ CNRS/LMD, Ecole Polytechnique, Palaison, France

\begin{abstract}
We present the measurement principle and the optical design of a Quad Mach Zehnder (QMZ) interferometer as HSRL technique, allowing simultaneous measurements of particle backscattering and wind velocity. Key features of this concept is to operate with a multimodal laser and do not require any frequency stabilization. These features are relevant especially for space applications for which high technical readiness level is required.
\end{abstract}

\section{INTRODUCTION}

Remote sensing technique such backscatter lidar has been used to derive aerosol and cloud layer boundaries and extinction. The retrieval of cloud and aerosol optical parameters requires in this case, clear air or use of the hypotheses with respect to the backscatter-to-extinction ratio. As identified in the early phase of lidar development, the high spectral resolution lidar (HSRL) technique allow the differentiation of molecular and particular scattering and more accurate characterization of aerosol or cloud extinction and backscatter coefficients. HSRL has the advantage of not requiring such inputs as molecular and aerosol particle scattering to be separated. Many HSRL systems have been already developed as filtering technique, by use of iodine filters, Michelson or Fabry-Perot interferometers (FPI), to separate the spectrally narrow aerosol scattering from the much broader molecular return. All of these systems operate in direct detection (measurement of the backscattered light power), which has the advantage of relying on both particulate and molecular scattering, allowing extended ranges and capabilities. The direct detection technique has been chosen for space observations of winds or aerosols and clouds using UV-FPI (ESA EarthCare mission). However, whatever the case, all the HSRL techniques developed until now required the emission of a quasi-monochromatic light, that is, the use of a single-mode laser, often injection seeded. Furthermore, most of the systems have also required precise frequency locking of the emitter with regard to the filters. These requirements lead to complex emitter designs that involve delicate servo-loops and can be points of failure in case of space applications.

In contrast, the Quadri-channel Mach Zehnder interferometer (QMZ) operates with a multimode laser. The optical path difference (OPD) of the QMZ matched to the laser free spectral range (FSR) so that the interference state of all the laser modes is exactly the same. The QMZ approach is not to separate the molecular and particulate signals in two distinct channels, but to determine the interference contrast given by the backscattered light that is linked to the backscatter ratio. The contrast is unambiguously determined by the signals given by four detection channels in phase quadrature, whatever the spectral positioning of the laser frequency with regard to the transmission function of the QMZ. In this way, no frequency stabilization is necessary neither for the laser nor the interferometer.

\section{METHODOLOGY}

\subsection{Measurement principle}

A schematic of a QMZ is shown in Figure 1. The main distinctive feature compared to a classical Mach Zehnder interferometer (MZI) is the introduction of quarter wave plate (QWP) in on arm of the interferometer, in order to introduce an 
additional phase difference of $\pi / 2$ for one direction of polarization with respect to the other. Assuming that no other polarization-dependent path difference occurs, the QMZ provides four output signals in phase quadrature. The two classical MZI output channels are separated by means of polarizers oriented along one axis of the QWP and the detection is made by four nonimaging detectors. In order to be operated with a multimode laser, the OPD, $\Delta$, of the QMZ must match the laser FSR such as

$$
\Delta=\frac{C}{F S R},
$$

where $c$ is the velocity of light in vacuum. In this case, the interference state at the QMZ output remains the same regardless the laser modes.

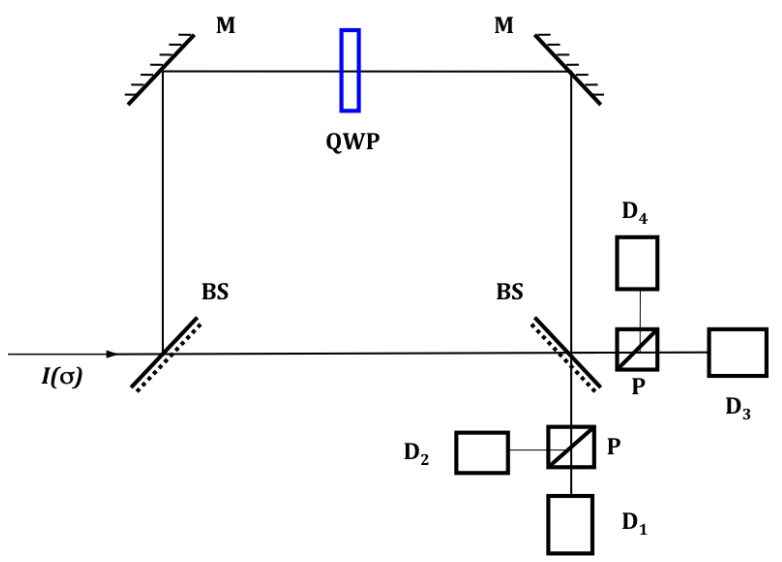

Figure 1 QMZ configuration: $M$, mirror; $B S$, beam splitter; $Q W P$, quarter-wave plate; $P$, polarizers; $D 1$ to D4, detectors

According to [1], with equal fractions of intensity transmitted through the polarizers, the signal delivered by each channel $i$ (from 1 to 4 ) is written as

$$
S_{i}=\frac{S_{t}}{4} a_{i}\left[1+M_{i} M_{a} \sin \left(\varphi+(i-1) \frac{\pi}{2}\right)\right] \text {, }
$$

where $S_{t}$ is the total signal, $a_{i}$ is the relative photometric sensitivity of channel $i, M_{i}$ is the instrumental interference contrast of channel $i, M_{a}$ is the interference contrast given by the atmospheric backscattered signal and $\varphi$ the interference phase. From Equation 2 we calculate the complex signal $Q$

$$
Q=Q_{2}+i Q_{1},
$$

with

$$
\begin{gathered}
Q_{1}=\frac{a_{3} S_{1}-a_{1} S_{3}}{a_{3} M_{3} S_{1}+a_{1} M_{1} S_{3}} \\
Q_{2}=\frac{a_{4} S_{2}-a_{2} S_{4}}{a_{4} M_{4} S_{2}+a_{2} M_{2} S_{4}} .
\end{gathered}
$$

The interference contrast given by the atmospheric backscattered signal is the modulus of the complex signal $Q$ :

$$
M_{a}=|Q| .
$$

Always according to [1], the atmospheric backscattered spectrum is the convolution of the emitted spectrum with the spectral broadening caused by the molecules and particles random motion. Therefore, the atmospheric contrast $M_{a}$ can be expressed as

$$
M_{a}=\frac{\beta_{p}}{\beta_{p}+\beta_{m}} M_{p}+\frac{\beta_{m}}{\beta_{p}+\beta_{m}} M_{m},
$$

Where $\beta_{p}$ and $\beta_{m}$ are the particle and molecule backscatter coefficients, respectively and $M_{p}$ and $M_{m}$ are the interference contrast given by particle and molecule coefficients, respectively.

The interference contrast given by a source of spectral full width at half maximum (FWHM), $\delta v$, is expressed according to [2] by:

$$
M=\exp \left[-\left(\frac{\pi \delta v \Delta}{2 c \sqrt{\ln (2)}}\right)^{2}\right]
$$

On the one hand, the spectrum backscattered by particles is spectrally broadened by wind turbulence in the probed volume and can be most of time neglected (a radial velocity of $1 \mathrm{~ms}^{-1}$ corresponds to a broadening of $4 \mathrm{MHz}$ at $532 \mathrm{~nm}$ ). The particle spectrum is then, as the laser spectrum, a comb of relatively narrow lines. The response of the laser-matched interferometer to this spectrum is exactly the same as for a single line of typical FWHM $\delta v \approx 200 \mathrm{MHz}$ and leads to an interference modulation $M_{p} \approx 0.8$ (Equation 7). On the other hand, the spectral broadening of the molecule-backscattered signal is much larger $(\approx 1$ $\mathrm{GHz}$ ) due to the wide molecular velocity distribution. The convolution with the emitted frequency comb of a typical $1 \mathrm{GHz}$ FSR produces a nearly constant spectral continuum without interference modulation $\left(M_{m} \approx 0\right)$. Thus, the resulting contrast is equal to the instrumental 
contrast produced by the laser source itself and the retrieved contrast, $M_{a}$, is equal to:

$$
M_{a}=R_{p} M_{p},
$$

with $R_{p}=\beta_{p} /\left(\beta_{p}+\beta_{m}\right)$ the particle backscatter ratio.

In addition to the contrast, the interference phase of the output signal is obtained by the argument of the complex signal $Q$

$$
\varphi=\arg (Q)=\frac{2 \pi \Delta}{\lambda_{0}}\left(1+\frac{2 V_{L O S}}{c}\right),
$$

where $\lambda_{0}$ is the emitted wavelength and $V_{L O S}$ the line-of-sight wind velocity. From the interference phase information we can retrieve the frequency of the backscattered signal and therefore the Doppler shift.

\subsection{Calibration and signal processing}

The calibration of the QMZ is based on the measurement of the two instrumental parameters: $a_{i}$ and $M_{i}$. These measurements can be done internally by means of a reference channel, consisting in collecting a small amount of light coming from the laser source. During atmospheric measurements, the complex signal is averaged over several laser shots in order to improve the measurement accuracy. As the interference phase may vary during this period, we still record the reference signal in order to perform a phasecompensated summation to avoid any underestimation of the contrast.

For a series of $\mathrm{N}$ shots, we have

$$
P B R=\frac{\beta_{p}}{\beta_{p}+\beta_{m}}=\frac{\sum_{1}^{N}\left|Q / Q_{R}\right|}{N},
$$

and

$$
V_{L O S}=\frac{c \lambda_{0}}{4 \pi \Delta} \arg \left(\sum_{1}^{N} Q / Q_{R}\right)
$$

where $Q_{R}$ is the reference complex signal.

\section{INSTRUMENT DESIGN}

The LATMOS laboratory has been developing for nearly a decade two QMZ interferometers. The first one is integrated to the French airborne lidar LNG, which is a 3 wavelengths and 2 polarizations backscatter lidar, dedicated to the aerosols and clouds studies [3]. The second one is part of the MARBLL lidar breadboard, which was designed with the aim of providing a compact and robust wind lidar for the Mars boundary layer study [2].

The design of the two QMZ devices is based on the same arrangement of fused silica prisms and air gap (Figure 2). In a general way, before entering the prism assembly the atmospheric signal is mixed with the reference signal with an unbalanced coupler $(90 \%$ for signal and $10 \%$ for reference). The atmospheric signal is previously delayed by a $60 \mathrm{~m}$ long fiber in order to be temporally separated from the reference. At the output of the coupler a mode scrambler is inserted in order to ensure good mode mixing and good depolarization.

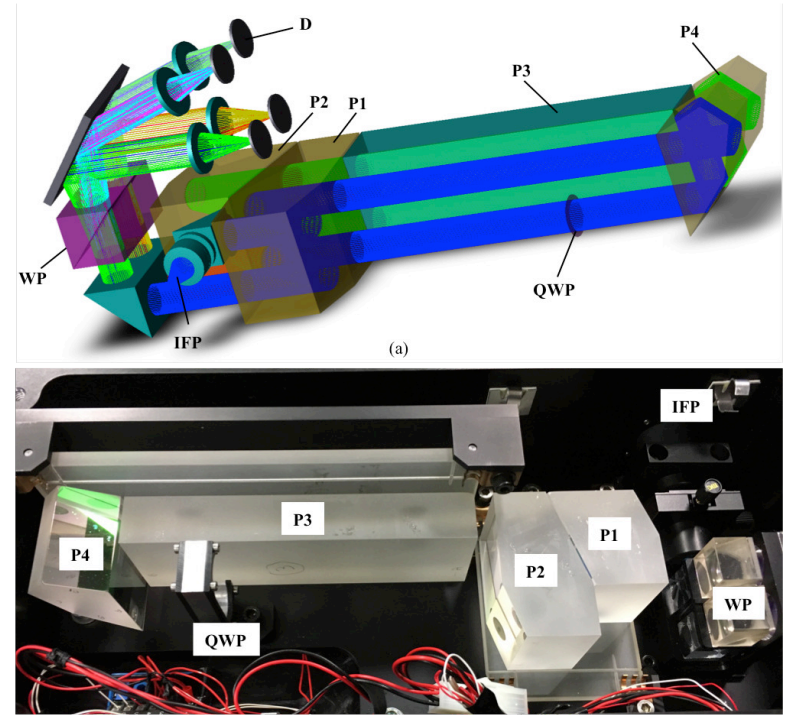

(b)

Figure 2 QMZ interferometer: (a) optical design of the QMZ, (b) picture of the MARBLL QMZ. P1, P2, P3,

$P 4$, prisms; IFP, input fiber port; $Q W P$, quarter-wave plate; WP, Wollaston polarizers; $D$, detectors.

At the entrance of the QMZ device, a first lens collimates the light. The first two prisms P1 and P2 enable the splitting and recombination of the two light paths with a nearly nonpolarizationsensitive $50 \%$ reflectivity coating. The first arm of the interferometer is made of a thick silica plate P3 and the second arm is an air gap in which the quarter wave plate is inserted. A second parallel pass in each arm is made possible by $\mathrm{P} 4$ that reflects the light in a laterally offset path. The two output ports of the interferometer are split in four channels by Wollaston polarizers (WP) and focused on the four detectors (D). The QMZ is field-compensated in order to fit large fiber 
diameter input without a reduction of the interference contrast.

We can note that the interferometer does not need any tuning element thanks to the high accuracy with which optical components are manufactured (by Winlight Optics, Pertuis, France).

\section{CONCLUSION}

Technical solutions for space-borne instrument must be the best compromise between scientific performances and technical readiness level (TRL). In terms of technical maturity, monomodal laser does not seem sufficiently high to envisage its deployment onboard space mission. In contrast, the high spectral resolution technique based on QMZ interferometer enable the use of a multimode laser. For instance, the MARBLL interferometer has been designed in order to be used with the same laser as the ChemCam instrument [4] currently in operation on Mars (Curiosity Rover). In addition, the QMZ solution does not require any frequency locking between the laser and the interferometer. In this way, the QMZ concept leads to a simpler and more robust design of the laser and lidar subsystems.

Regarding the measurement performances and when considering particle backscattering lidar, Equation 8 shows that the measurement method is independent of the exact molecular line-width. This avoids the need of precise line-shape modeling based on pressure and temperature profiles, as required for the HSRL vapor-filter and FPI techniques. Note also that the PBR retrieval is not affected by a Doppler shift of the signal, which could be caused by the platform motion and pointing. This last point is a non-negligible advantage for space-borne instrument. Bruneau et al. (2015) [3] describe in details the first results of the $355 \mathrm{~nm}$ high spectral resolution airborne lidar LNG and also [5] describe results of the MARBLL first field campaign at the Observatoire de Haute-Provence in France.

In the coming months, thermal vacuum tests will be carried out on the MARBLL QMZ breadboard in order to control the measurement stability over large temperature variations under vacuum (from $20^{\circ} \mathrm{C}$ to $+40^{\circ} \mathrm{C}$ ). The final purpose of these tests is to increase the maturity level of the QMZ concept by moving from TRL 3 to TRL 4 .
At last, in preparation of phase 0 of the FrenchAmerican MESCAL space-borne project, involving the Centre National d'Etudes Spatiales (CNES), the National Aeronautics and Space Administration (NASA) and Airbus Defence and Space Company (ADS), the LATMOS laboratory has been identified to provide a conceptual study and a prototype of the $532 \mathrm{~nm}$ HSRL channel based on the QMZ concept. This project, which could take over from the current CALIPSO mission, will be devoted to the study of the climate evolution by measuring clouds and aerosols in the Earth atmosphere.

\section{ACKNOWLEDGEMENTS}

We thank the French Space Agency CNES for their continuous support for the development of MARBLL and the phase 0 of MESCAL project.

\section{References}

[1] Bruneau, D., Pelon, J., Simultaneous measurements of particle backscattering and extinction coefficients and wind velocity by lidar with a Mach-Zehnder interferometer: principle of operation and performance assessment, Appl. Opt. 42, 1101-1114 (2003)

[2] Bruneau, D., et al., Direct-detection wind lidar operating with a multimode laser, Appl. Opt. 52, 4941-4949 (2013)

[3] Bruneau, D., et al., 355-nm high spectral resolution airborne lidar LNG: system description and first results, Appl. Opt. 54, 8776-8785 (2015)

[4] Maurice, S., Wiens, R.C., Saccoccio, M. et al. Space Sci Rev (2012) 170: 95.doi:10.1007/s11214-012-9912-2

[5] Määttänen, A., et al., Towards a Martian wind lidar, 2017, ILRC 2017, Bucarest, Romania 